\title{
The Physical Design of Biological Systems - Insights from the Fly Brain
}

\author{
Louis K. Scheffer \\ schefferl@janelia.hhmi.org \\ Janelia Research Campus \\ Howard Hughes Medical Institute \\ Ashburn, Virginia, USA
}

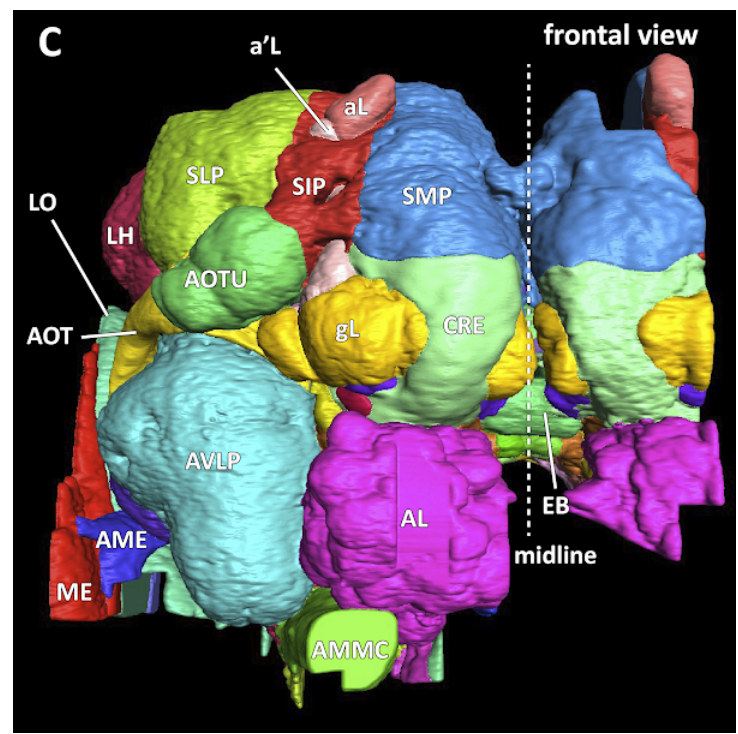

(a) Brain regions

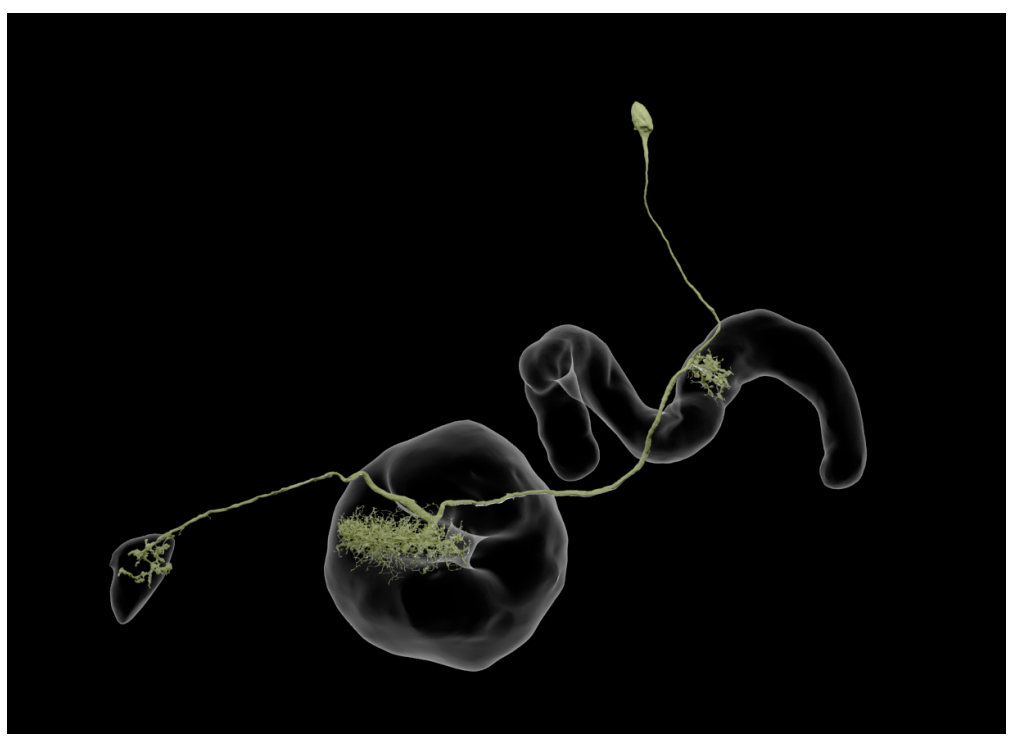

(b) A single neuron. The semi-transparent volumes are two of the regions shown in panel (a)

Figure 1: Overview of fly brain reconstruction. Adapted from [22].

\begin{abstract}
Many different physical substrates can support complex computation. This is particularly apparent when considering human made and biological systems that perform similar functions, such as visually guided navigation. In common, however, is the need for good physical design, as such designs are smaller, faster, lighter, and lower power, factors in both the jungle and the marketplace. Although the physical design of man-made systems is relatively well understood, the physical design of biological computation has remained murky due to a lack of detailed information on their construction. The recent EM (electron microscope) reconstruction of the central brain of the fruit fly now allows us to start to examine these issues. Here we look at the physical design of the fly brain, including such factors as fan-in and fanout, logic depth, division
\end{abstract}

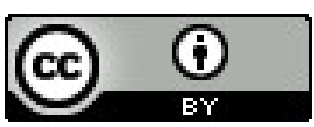

This work is licensed under a Creative Commons Attribution International 4.0 License.

ISPD '21, March 22-24, 2021, Virtual Event, USA.

(C) 2021 Copyright is held by the owner/author(s).

ACM ISBN 978-1-4503-8300-4/21/03.

https://doi.org/10.1145/3439706.3446898 into physical compartments and how this affects electrical response, pin to computation ratios (Rent's rule), and other physical characteristics of at least one biological computation substrate. From this we speculate on how physical design algorithms might change if the target implementation was a biological neural network.

\section{CCS CONCEPTS}

- Computer systems organization $\rightarrow$ Neural networks; Analog computers; Self-organizing autonomic computing; Molecular computing;

\section{KEYWORDS}

brain architecture; neuron operation; physical design

\section{ACM Reference Format:}

Louis K. Scheffer. 2021. The Physical Design of Biological Systems - Insights from the Fly Brain. In Proceedings of the 2021 International Symposium on Physical Design (ISPD '21), March 22-24, 2021, Virtual Event, USA. ACM, New York, NY, USA, 8 pages. https://doi.org/10.1145/3439706.3446898

\section{INTRODUCTION}

Good physical design is an essential requirement of both humanmade and biological systems. Both have huge incentives to pack 
their computation as tightly as possible. A tighter packing of the same computation yields faster operation, lower energy consumption, less material cost, and lower mass. For biological systems, a good physical design is literally a matter of life and death.

We cannot currently design a brain to perform any specific function, in large part because we do not yet understand how the brain performs the functions it has, though it's been obvious for decades that from a purely physical design perspective, their details and methods are quite different. However, as our understanding of the physical construction[22] and operation([11][27] and many others) of the brain increases, we can begin to speculate on how physical design, as covered by this conference, might change if the target implementation technology was biological.

This article first summarizes what we know of the physical construction of the fly brain, and how it operates. Even from this murky perspective, it is clear that many of the topics covered by this conference would need to be addressed differently to perform the physical design of a biological system. We summarize some of these differences and conclude with a look forward.

\subsection{Characteristics of the fly brain}

The brain is fully three dimensional as opposed to the interconnected $2 \mathrm{D}$ assemblies of human designs.

1.1.1 Neurons vs gates and wires. In human-designed systems, the 'gates' do the computation, and are connected by 'wires' which only communicate. In a biological system a single component type, a 'neuron' does both.

The neuron does not perform the simple boolean operations typical of gates in human-designed systems. Instead, most neurons perform an operation called a "leaky integrate and fire", where weighted inputs are integrated by a leaky integrator, and if the instantaneous voltage exceeds a threshold, the cell fires, generating an output spike, and then resets. However, there are also cells that produce "graded potentials", a non-linear summation of their inputs without generating spikes. There are even cells that change their mode of operation dynamically - for example, linear summation during the day, but non-linear with spiking at night[20]. A final complication is that neurons can simultaneously perform different operations in sufficiently separated branches[18].

1.1.2 Non-local communication. Another big difference is that human designers go to a great deal of trouble to ensure the activity of one wire or gate does not affect the activity of other constructs nearby. This goal underlies the physical design fields of 'crosstalk control' and 'power grid design'. Nerve cells, however, embrace non-local effects. Chemical synapses and gap junctions (resistive connections between cells) rely on physical proximity, similar to electrical gates. But nerve cells also communicate by neuromodulators, which work by diffusion and have longer ranges of action. For example, for dopamine[21], a typical neuromodulator, a less sensitive receptor might have a range of 2 microns, where a more sensitve receptor may have a range of 7 microns. As shown in Figure 2 this covers a substantial number of neurons. Human-designed systems, and the placement and routing algorithms that design them, do not currently support these longer range, but slower, connections.

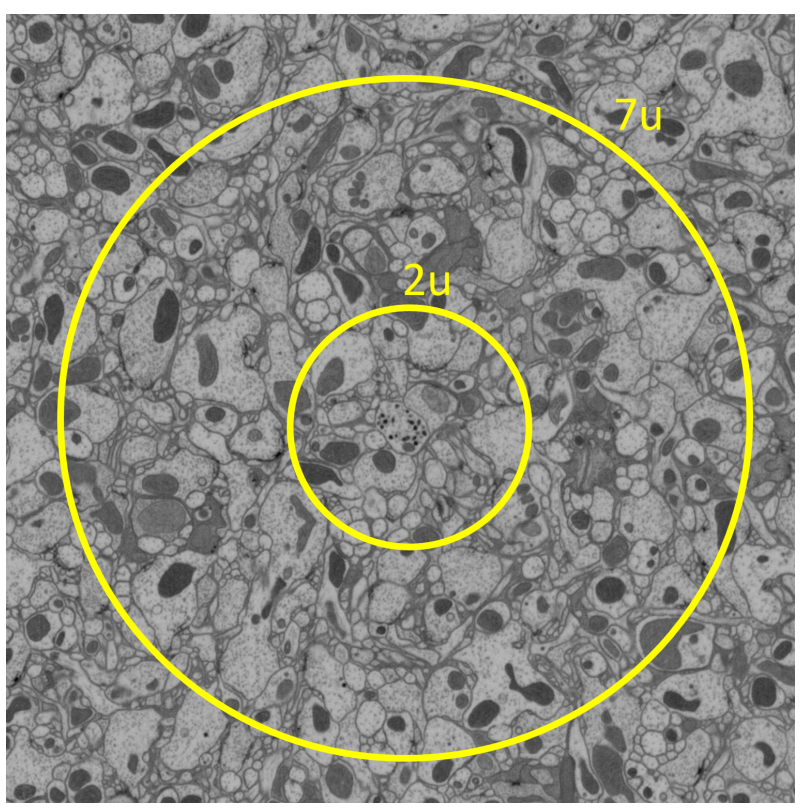

Figure 2: Image showing range of neuromodulators

1.1.3 Clock and power networks. Power is delivered in chemical form, mostly by diffusion in the fly. Interlaced with the neurons there are support cells called 'glia', which are thought to help with energy acquisition, waste disposal, and other housekeeping requirements. However their precise role remains poorly understood.

There are no high speed clocks; the fly's circuits are largely asynchronous. Circadian clock (one cycle per day) circuitry is present, but operates slowly so clock skew is not an issue.

1.1.4 Design for manufacturability and yield. High fanout and analog operation give graceful degradation. Furthermore, biological circuits are typically redundant, presumably to cope with cell death, injury, errors in development, and other factors. Indeed, the circuits are typically so redundant that experiments creating 'stuck at' faults often have little observable effect[31].

1.1.5 Placement and Routing. The process that generates the final placement and routing in the brain is a "precisely orchestrated sequence of genetic, environmental, biochemical, and physical events"[3], all directed by selective expression of components of the genome. We are currently very far from being able to construct, de novo, a genome that creates a specified placement and routing.

Some factors that determine the placement and wiring are beginning to be understood. Placement and global routing are determined by some combination of growing cells following gradients and physical competition for space, with the whole process taking place in three-dimensions. Detailed routing is thought to be determined by some combination of surface proteins expressed by cells, and their mutual activity ("Cells that fire together, wire together")[14].

1.1.6 Fanouts are large and paths are short. The fan-out and fan-in of neurons are much larger than that of gates. A typical neuron in the fly brain has a fan-in and fan-out of 143 (the two are necessarily equal when averaged over the whole brain). 

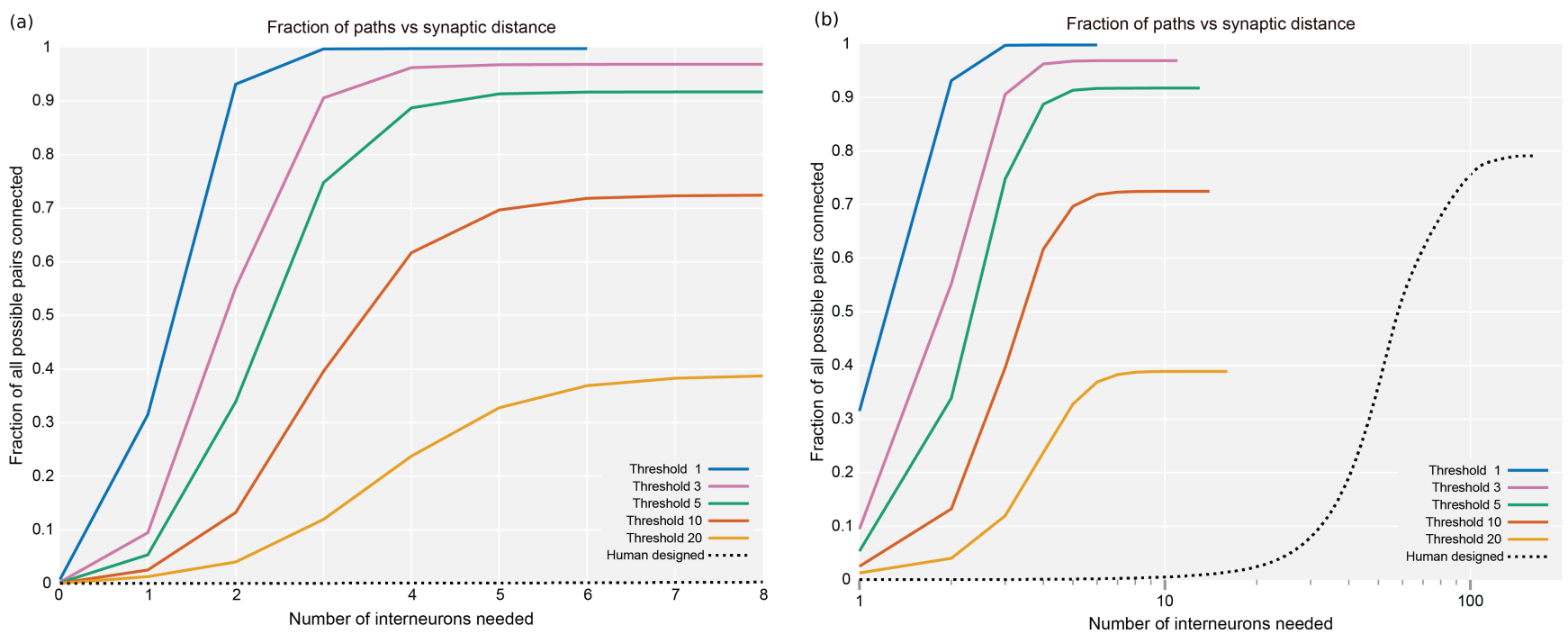

Figure 3: Plots of the percentage of pairs connected (of all possible) versus the number of interneurons required. (a) shows the data from the whole hemibrain, for up to 8 interneurons. (b) is a much wider view of the same data, shown on a log scale so the curve from a human designed system is visible. From [22].

The high fanout results in very short average path lengths, as shown in Figure 3, which plots what fraction of neuron pairs are connected as a function of the number of interneurons between them. Three quarters of all possible pairs are connected by a path with fewer than three interneurons, even when only connections with $\geq 5$ synapses are included. If weaker connections are allowed, the paths become shorter yet. These short paths and tight coupling are very different from human designed systems, which have much longer path lengths connecting node pairs. As an example, a standard electrical engineering test circuit (S38584 from the ISCAS '89 benchmark suite[2]) is shown alongside the hemibrain data in Figure $3 \mathrm{~A}-\mathrm{B}$. The connection graph for this example has roughly the same number of nodes as the graph of the fly brain, but pair-to-pair connections involve paths more than an order of magnitude longer - a typical node pair is separated by 60 intervening nodes. This is because a typical computational element in a human designed circuit (a gate) connects only to a few other elements, whereas a typical neuron receives input from, and sends outputs to, hundreds of other neurons.

1.1.7 Low power utilization. Small insects necessarily use very little power, yet perform significant computation, and have done so for hundreds of millions of years[8]. Efficient operation is at a premium for these animals, as each joule used for computation has to be obtained by risky foraging, and cannot be used for other demands such as seeking mates or avoiding predators. Therefore evolution, over the course of hundreds of millions of years, has honed these circuits to work on a small power budget.

The power budget of an animal can be determined from respiration rates. Chadwick[5] cites oxygen consumption of a resting fly as $26 \mathrm{~mm}^{3} / \mathrm{gram} /$ minute, with a typical fly mass of $1.0 \mathrm{mg}$ (these were large Drosophila species). This gives $2.6 \cdot 10^{-2} \mathrm{~mm}^{3} /$ minute or $2.6 \cdot 10^{-8} \mathrm{l} / \mathrm{min}$. But one liter of $\mathrm{O}_{2}$ metabolizes to about $5 \mathrm{KCal}$ or about $2 \cdot 10^{4}$ joules. So we get $5.2 \cdot 10^{-4}$ joule/minute, or about $10^{-5}$ joules/sec, or watts. But Drosophila melanogaster are about $1 / 4$ the weight of the measured flies, and only about $5 \%$ of the resting metabolism of flies is thought to be used by the nervous system[19]. So the computing power is thought to be about $1.2 \cdot 10^{-7} \mathrm{w}$, or $120 \mathrm{nw}$, for Drosophila melanogaster, from which the circuits below were derived.

This seems economical when compared to energy use in other insect cells, though there are not many measurements. Wagner[32] reports a consumption in 'Sf9' cells of about 33 atto-moles/sec of $\mathrm{O}_{2}$, which translates to about $1.55 \cdot 10^{-11} \mathrm{w} /$ cell, which would correspond to about $1.55 \cdot 10^{-6} \mathrm{w}$ for the $10^{5}$ cells of the Drosophila brain. This is more than is observed in the resting Drosophila above, but it is known that brain responses (and hence presumably power consumption) are faster in active states such as walking[6]. Combining these lines of evidence, it appears Drosophila achieves its low power operation by starting with relatively low power cells, using a circuit organization and architecture with a minimum number of cells, and perhaps power switching depending on state.

1.1.8 Low voltage operation. One of the main ways biology keeps power dissipation down is low voltage operation. In the nervous system of Drosophila, and other animals, the biggest voltage swings are about $60 \mathrm{mv}$, as opposed to human low power systems where the voltage swings are roughly an order of magnitude higher. Since the power consumed in a single logic transition scales as $C \cdot V^{2}$, biological systems have a roughly $100 \mathrm{x}$ advantage in power per transition. Graded (analog) neurons use even smaller voltages swings, offering even more potential power savings.

Conventional MOS electronics cannot easily use such small swings, since $k T / q$ (where $k$ is Boltzman's constant, $T$ is the temperature, and $q$ is the charge of the gating element, in CMOS a single electron) is about $25 \mathrm{mv}$ at room temperature. Therefore devices 
(a)

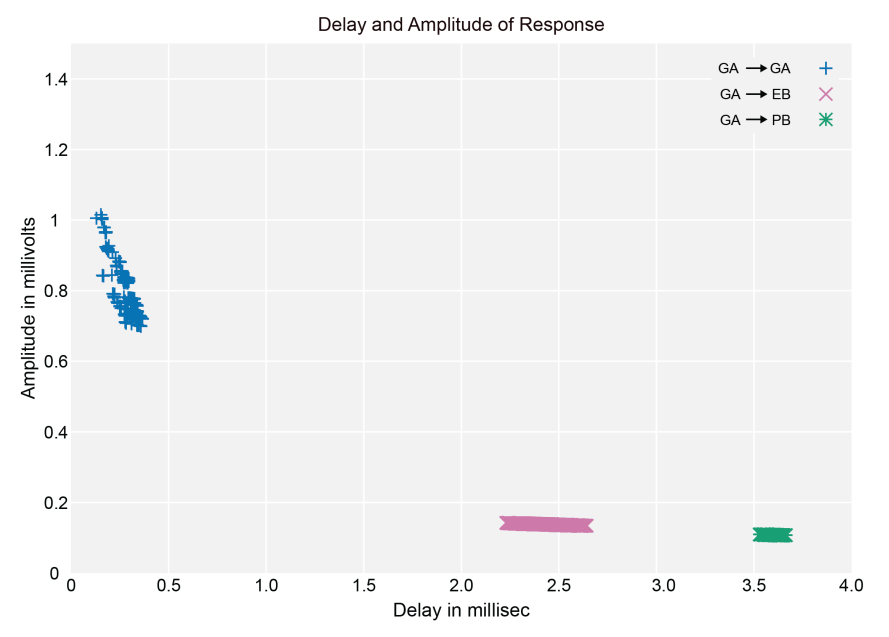

(b)

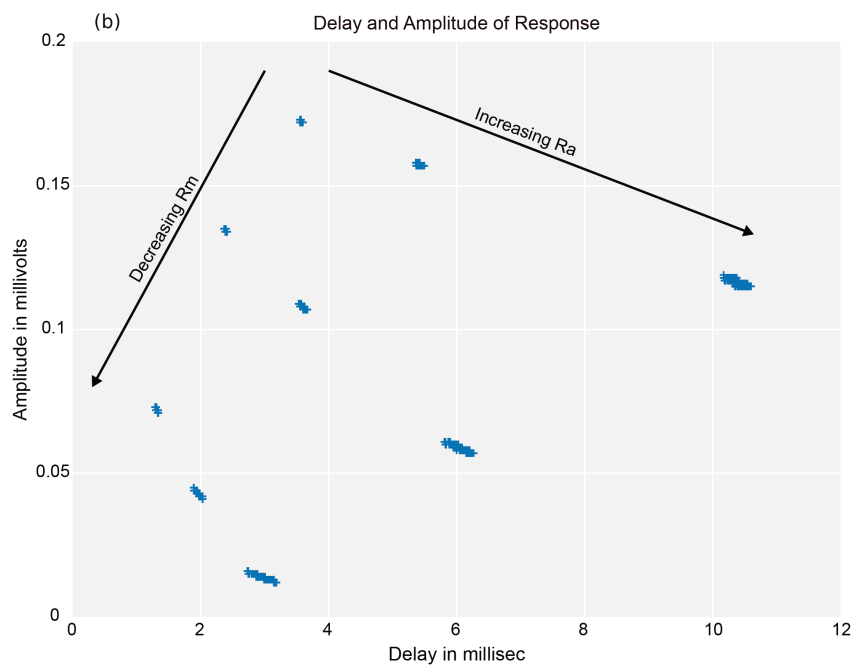

Figure 4: (a) The linear response to inputs in the gall(GA) for an EPG neuron, which also has arbors in the ellipsoid body(EB) and the protocerebral bridge (PB). Each point in the modeled plot shows the time each response reached its peak amplitude (the delay), and the amplitude at that time, for an input injected at one of the PSDs in the Gall. (b) Delays and amplitudes for gall to $\mathrm{PB}$ response, for all combinations of three values of cytoplasmic resistance $R_{A}$ and three values of membrane resistance $R_{M}$. From [22].

based on the movement of individual electrons will have a change $\Delta I=e^{\Delta V /(k T / q)}$, leading to the canonical decade change in current for each $60 \mathrm{mv}$ change in input voltage. In MOS electronics, this implies that a very small voltage swing leads directly to a limited on-off ratio, and hence high static power consumption. This very fundamental line of reasoning was worked out in 1972[26], at the very beginning of the CMOS era, and limits the minimum voltage swing to about $8 k T / q$, or about $200 \mathrm{mv}$. This ideal limit must be further increased to account for threshold variation.

How then do nerve cells operate with lower voltages? Perhaps surprisingly (to EEs, anyway) this was worked out even earlier (in 1952) in the Nobel prize winning research of Hodgkin and Huxley [12]. Looking at the slope of current vs voltage of voltage-gated ion channels, they used exactly the same line of reasoning to determine that at least 5 or 6 electron charges must be involved to open or close an ion channel. This directly reduces the value of $k T / q$ and allows biological voltage swings to change currents by many orders of magnitude. The tradeoff is slower operation, since many individual events need to take place to change the status of a "switch".

Furthermore, biological voltage-controlled switches (ion channels) are defined by chemical molecules, not by bulk properties such as doping density or oxide thickness. So unlike CMOS, each "device" has the same threshold, and extra switching voltage is not needed to account for manufacturing variation.

In addition, biological circuits are normally relatively insensitive to transient errors in their inputs. For example, many neurons work on the principle of integrating their inputs, using current sources implemented by these ion channels. Inadvertent thermal activation of input channels will normally be short term and have little consequence.
Finally, the biological circuits are typically redundant, presumably to cope with cell death, injury, errors in development, and other factors. For such circuits an occasional spurious activation has little effect.

1.1.9 Timing optimization. The basic techniques used in the fly brain to reduce response time are short path lengths, parallelism, and the physical design of important paths. For example, the 'giant fiber', important in escape reflexes, has a large diameter since axial resistance goes down like the square of diameter, whereas $\mathrm{C}$ grows only linearly, so the RC product is less for larger diameter lines.

One technique used in larger animals, myelinated axons, is not used in the fly brain.

1.1.10 Compartmentalization predicts delay and amplitude response. How does the compartmentalization of the fly brain affect neural computation? In a few cases this has been established. For example, the CT1 neuron performs largely independent computations in each branch[18], whereas estimates show that within the medulla, the delays within each neuron are likely not significant for single column optic lobe neurons, and hence the neurons likely perform only a single computation[28]. Similarly, compartments of PEN2 neurons in the protocerebral bridge have been shown to respond entirely differently from their compartments in the ellipsoid body[9][30].

Detailed skeleton models from EM reconstruction allow the construct of electrical models of neurons. (In what follows, we use the word 'compartment' to mean a named physical region of the brain, as shown in Figure 1(a), as opposed to the electrical sub-divisions used in simulation.) In particular, to look more generally at the issues of intra- vs inter-compartment delays and amplitudes, we can construct a linear passive model for each neuron. Our method is similar to that elsewhere[23], except that instead of using right 
cylinders, we represent each segment of the skeleton as a truncated cone. This is then used to derive the axonic resistance, the membrane resistance, and membrane capacitance for each segment. To analyze the effect of compartment structure on neuron operation, we inject the neuron at a postsynaptic density (input) with a signal corresponding to a typical synaptic input ( $1 \mathrm{nS}$ conductance, $1 \mathrm{~ms}$ width, $0.1 \mathrm{~ms}$ rise time constant, $1 \mathrm{~ms}$ fall time constant, $60 \mathrm{mV}$ reversal potential). We then compute the response at each of the T-bar sites (outputs). Since the synapses, both input and output, are annotated by the brain region that contains them, this allows us to calculate the amplitudes and delays from each synapse (or a sample of synapses) in each compartment to each output synapse in all other compartments.

In general, we find the compartment structure of the neuron is clearly reflected in the electrical response. Consider, for example, the EPG neuron (Figure 1(b)) with arbors in the ellipsoid body, the protocerebral bridge, and the gall (the gall is a sub-compartment of the LAL, the lateral accessory lobe). Figure 4(a) shows the responses to synaptic input in the gall. Within the gall, the delays are very short, and the amplitude relatively high and variable, depending somewhat on the input and output synapse within the gall. From the gall to other regions the delays are longer (typically a few milliseconds) and the amplitudes much smaller and nearly constant, largely independent of the exact transmitting and receiving synapse. There is a very clean separation between the within-compartment and across-compartment delays and amplitudes, as shown in Figure 4(a). The same overall behavior is true for inputs into the other regions - short delays and strong responses within the compartment, with longer delays and smaller amplitudes to other compartments.

This simple pattern motivates a model that describes delays and amplitudes not as a single number, but as an $N \times N$ matrix, where $N$ is the number of compartments. Each row contains the estimated amplitude and delay, measured in each compartment, for a synaptic input in the given compartment. This gives a much improved estimate of the linear response. For the example EPG neuron above, with nominal values for $R_{a}, R_{m}$, and $C_{m}$, if we represent all delays by a single number then the standard deviation of the error is 0.446 ms. If instead we represent the delays as a $3 \times 3$ matrix indexed by the compartment, the average error is $0.045 \mathrm{~ms}$, for $10 \mathrm{x}$ greater accuracy. Similarly, the average error in amplitude drops from 0.168 $\mathrm{mv}$ to $0.021 \mathrm{mv}$, an eightfold improvement. While the improvement in error will depend on the neuron topology, in all cases it will be more accurate than a point model, for relatively little increase in complexity.

The absolute values of delay and amplitude are strongly dependent on the electrical parameters of the cell, however. A wide range of electrical properties has been reported in the fly literature (see Table 5 in [22]) and it is plausible that these vary on a cell-to-cell basis. In addition gap junctions, which are not included in our model, could affect the apparent value of $R_{m}$. In light of these uncertainties, we simulate with minimum, medium, and maximal values of $R_{a}$ and $R_{m}$, for a total of 9 cases, as shown in Figure 4(b). All are needed since the resistance parameters interact non-linearly. We fix the value of $C_{m}$ at $0.01 \mathrm{~F} / \mathrm{m}^{2}$ since this value is determined by the membrane thickness and is not expected to vary from cell to cell[15]. The results over the parameter range are shown in Figure 4(b) for the case of the EPG neuron above for delay from the gall to

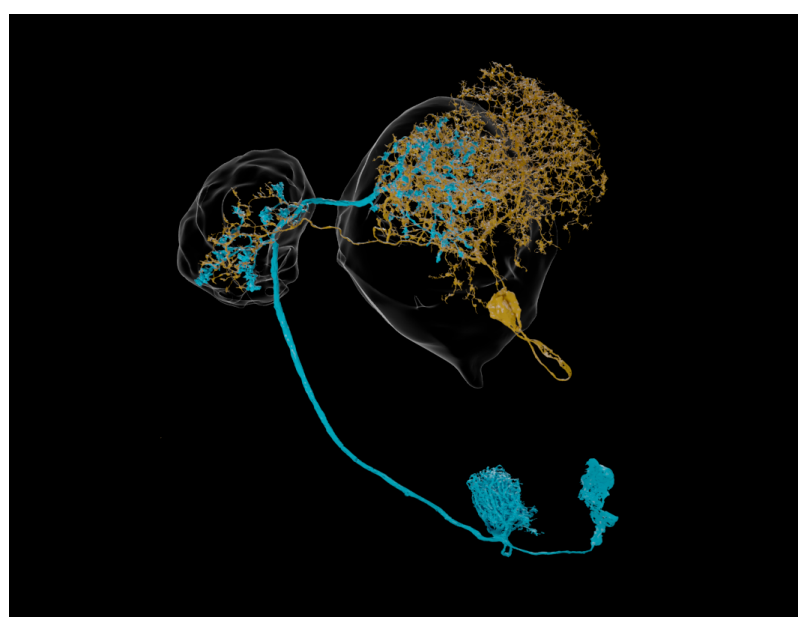

Figure 5: Two neurons that connect in more than one compartment, in this case the calyx and the lateral horn. They are each pre- and postsynaptic to each other in both compartments. From [22].

the $\mathrm{PB}$. The intra-compartment and between-compartment values are well separated for any value of the parameters (not shown).

Programs that deduce synaptic strength and sign by fitting a computed response to a connectome and measured electrical or calcium imaging data[29] may at some point require estimates of the delays within cells. If this is required, the above results suggest this could be accomplished with reasonable accuracy with a compartment-to-compartment delay table and 2 additional parameters per neuron, $R_{A}$ and $R_{M}$. This is relatively few new parameters in addition to the many synaptic strengths already fitted.

A number of neurons have parallel connections in separate compartments (see Figure 5). This motif is common in the fly's brain - about $5 \%$ of all connections involving 6 or more synapses are spread across two or more non-adjacent compartments. Given the increased delays and lower amplitudes of cross-compartment responses, this type of interaction differs electrically from those in which all connections are contained in a single compartment. A point neuron model cannot generate an accurate response for such connections - a synapse in region A will result in a fast response in $A$ and a slower, smaller response in $\mathrm{B}$, and vice versa, even though both of these events involve communication between the same two neurons. It is not known if this configuration has a significant influence on the neurons' operation.

From these models we conclude (a) the compartment structure of the fly brain shows up directly in the electrical response of the neurons, and (b) the compartment structure, though defined anatomically, matches that of the electrical response. From the clear separation in Figure 4, it is likely that the same compartment definitions could be found starting with the electrical response, though we have not tried this. (c) These results suggest a low dimensional model for neural operation, at least in the linear region. A small region-to-region matrix can represent the delays and amplitudes well. (d) Absolute delays depend strongly (but in a very predicable manner) on the values of axial and membrane resistance, which can 


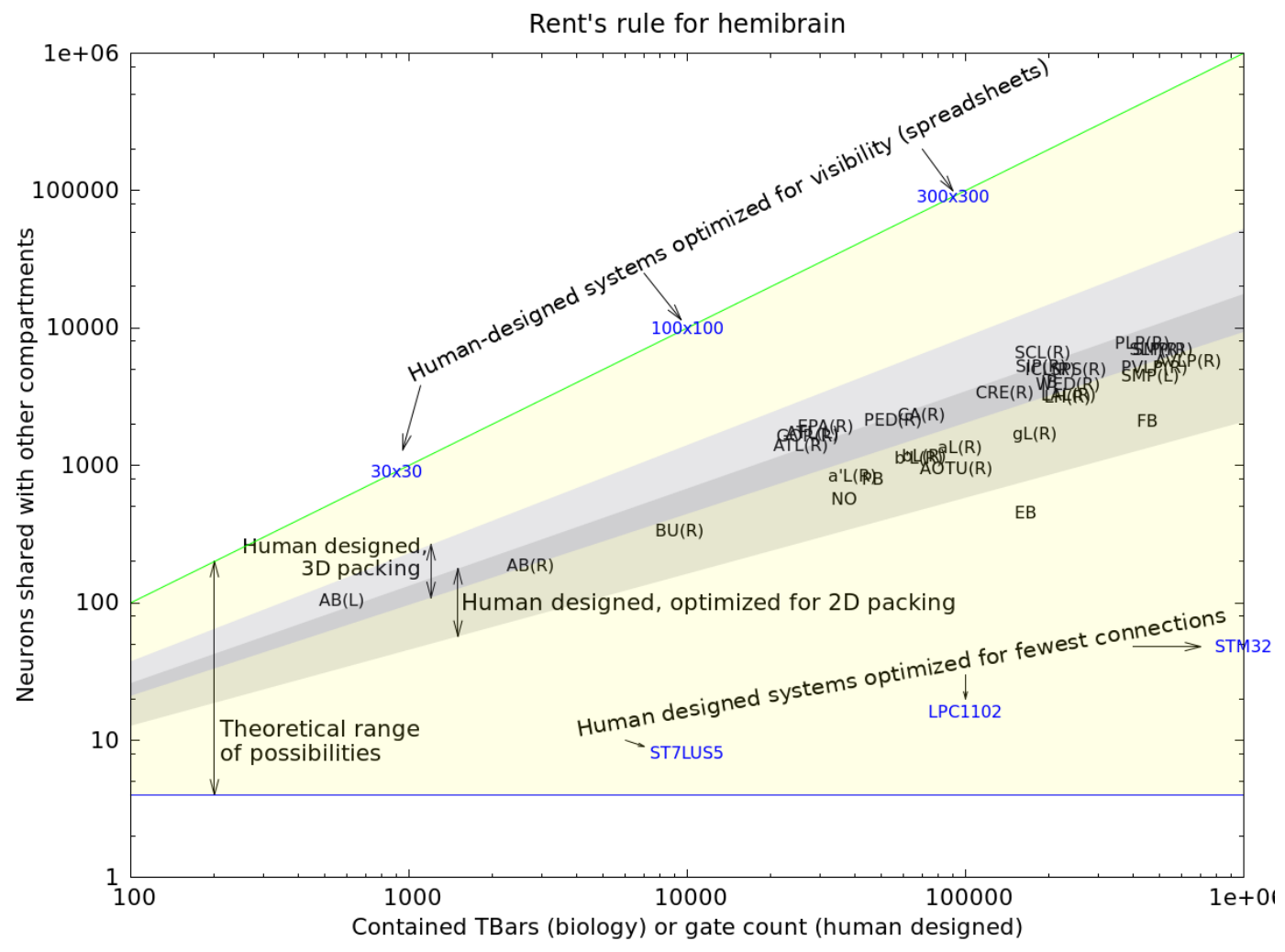

Figure 6: Rent's rule for the central brain of the fruit fly. The yellow region encompasses theoretical bounds for computation. Four varieties of human-designed systems are shown. Those designed for visibility into computation achieve the upper bound, while those designed for minimum communication approach the lower bounds (Microprocessors ST7LU55, LPC1102, and STM32). Human designed systems where efficient packing is the main criterion occupy the shaded area (in 2D and 3D). The characteristics of the primary fly-brain compartments that are completely contained in the reconstructed volume are shown with alphanumeric labels, such as "AB(R)" or "EB". (See [13] for compartment definitions.) The hemibrain compartments fall very nearly in the same range as human designed systems designed for efficient packing. Data from [22].

vary both from animal to animal and from cell to cell. (e) Neurons that have parallel connections in separate compartments have a different electrical response than they would have with the same total number of synapses in a single compartment.

1.1.11 Partitioning. Partitioning of a physical system into smaller components looks similar in human and biological systems. One exception, however, is that human designed systems typically segregate computation and memory, whereas in biological systems these are mixed in a manner that is not well understood. However, despite their differences, the overall interconnections requirements between partitions looks similar.

Rent's rule[17], well known to EEs, is an empirical observation that when a human designed system is packed as tightly as possible, at every level of the hierarchy the required communication (the number of pins) scales as a power law of the amount of contained computation, measured in gates. As an observed relationship, Rent's rule is not derived from underlying theory, and the relationship is not exact and still contains scatter. Biological equivalents to this rule exist, such as the observation that brain size tends to vary as a power law of body size[10], across a wide range of species occupying very different ecological and behavioral niches. Rent's rule is roughly true over many orders of magnitude in scale, and for almost every system in which it has been measured. Somewhat surprisingly, Rent's rule applies almost independently of the function performed by the computation being performed, and at every level of a hierarchical system. It also applies whether the compactness criterion is minimization of communication (partitioning) or physical close packing.

Rent's rule is expressed as

$$
\text { Pins }=a *(\text { computation })^{b}
$$

where $a$ is a scale factor (typically in the range 1-4), and $b$ is the 'Rent exponent' describing how the number of connections to the compartment varies as a function of the amount of computation performed in the compartment. The Rent exponent has a theoretical range of 0.0 to 1.0 , where 0 represents a constant number of connections, with no dependence on the amount of computation performed, and 1.0 represents a circuit in which every computation is visible on a connection. Human designed computational systems 
occupy almost the full range, from spreadsheets in which all intermediate results are visible, to largely serial systems in which minimizing communication (pins) is critical. This relationship is shown in Figure 6. However, when the overriding criterion is that the system must be packed as tightly as possible, Rent observed that the exponent of the power law falls in a close range of roughly 0.5-0.7.

For electrical circuits, the computation is measured in gates, and the connections are measured by pin count. These ranges are shown in Figure 6 for circuits that are roughly the size of the fly's brain, packed in either two[33] or three[7] dimensions.

Also shown in this plot are the values for the fly's brain computational regions. In this case, the computation is measured as the number of contained T-bars (the transmitting side of the synapse in the fly), and the connection count is the number of neurons that have at least one synapse both inside and outside the compartment. (Very similar results are obtained if the computation is measured as the number of signal receptors, or the number of unique connection pairs). The sub-divisions used in the calculation are the compartments of the fly brain, as defined by anatomists[13], and shown in Figure 1(a). Almost all the fly brain compartments fall well within the range of exponents expected for packing-dominated systems, while the ellipsoid body (EB) falls just outside the expected area. This is perhaps due to the large number of strongly connected clique-containing circuits in the ellipsoid body[22] since such circuits have relatively few connections for the amount of synapses they contain.

As noted, a tighter packing of the same computation yields faster operation, lower energy consumption, less material cost, and lower mass. A natural speculation, therefore, is that both the humandesigned and evolved systems are dominated by packing considerations, and that both have found similar solutions.

1.1.12 Hardware security-related physical design. Somewhat surprisingly, this is a real problem, as examples of insect brain hijacking[1] show. As far as we know, no design techniques are used to guard against this. Instead insects use physical defenses, such as a hard carapace and a blood-brain barrier, and behavioral modifications, to avoid problems such as this.

\section{IMPLICATIONS FOR PHYSICAL DESIGN}

Here we look at the implications for physical design algorithms, when we get that far. Much of this is speculative since we do not yet have a solid understanding of how biological systems are built and operate.

2.0.1 Floorplanning and interconnect planning. The floorplan of the insect brains has changed little over millions of years[25]. It is very difficult for biology to evolve a distinctively different floorplan since any design must be evolved by a series of minor changes, each of which must be competitive with the best existing solutions. It is completely unknown how close the existing floorplans are to optimum - an interesting experiment might be to run the fly connectome through known 3D floorplanning tools. Given that Rent's rule holds for both, it is possible they would be of similar quality, but interesting no matter how it turns out.
2.0.2 Partitioning. Partitioning in human designs and biological designs appears similar. This is likely due to good packing being an important component of each. A likely (but unproven) consequence is that partitioning algorithms developed for human designs would likely work well for biological designs, with relatively minor changes to accomodate larger fanouts and non-local signals.

2.0.3 Placement, and routing. We need considerably more understanding before we can implement placement and routing algorithms for biological systems.

2.0.4 Physical synthesis. In biological systems, almost all functional changes are similar to what human systems call ECO (engineering change order) mode[16] in synthesis - only small changes to an already working design are allowed. It is thought that even major changes are implemented by a sequence of duplication and then divergence, as each intermediate version must not only work, but must be competitive with existing designs[24].

The next step in physical synthesis of biological systems would be synthesizing an implementation, either logical or physical, from a higher level logic or behavioral description. Most likely this would be a combination of synthesis of asynchronous systems [4] built from threshold gates[34], with the additional (strong) constraint that the interconnect pattern be implementable by a short genetic algorithm. This would be among the most changed algorithms, as it would need to account for large fanout, short usable paths, and very different logical operation.

Also needed would be new template libraries, with known good implementations of needed functions. At least initially, this would likely be based on existing circuits. It is currently not known how large such a library would be - there are millions of species, but they share an enormous amount of biological heritage. The neural logic of many more creatures will need to be examined before even the outlines of this library emerge.

2.0.5 Machine learning for physical design. Biological designs are subject to many simultaneous constraints of different kinds. This could make classical algorithms less useful, as they tend to optimize a single objective. Machine learning could potentially offer a way around this. Given enough examples, machine learning could potentially find good ways to combine known structures to perform desired functions. However, getting enough examples may be difficult, since currently reverse engineering biological circuits is expensive and time consuming.

2.0.6 Interactions with system and logic level design. In the insect brain, placement and routing are systematically intertwined with system and logic level design, and the performance of the resulting system. Evolution, the "designer" of the fly brain, tweaks all of these (and more) in parallel. Algorithms considering physical design only will likely not be competive in this space.

2.0.7 Timing driven design. In biology, individual logic elements are slow (ms compared to ns for human-designed logic), so minimization of logic levels is critical. The physical characteristics of neurons can also be adjusted to some extent. Meeting a timing goal will likely require a combination of these techniques.

2.0.8 Hardware security-related physical design. This is a fascinating but very speculative possibility. For example, if nervous systems 
could be designed, presumably they could incorporate mathematical forms of error detection and correction that would be unlikely to evolve organically.

\section{CONCLUSIONS}

Physical design for biological systems is a ways out, but as we begin to understand the working of existing biological examples, the broad outlines are slowly taking shape. Some existing physical design tools will be unneeded, such as low skew clock design. Some will be similar to existing tools, such as partitioning. Some will be based on known technologies, such as delay calculation. Some will be based on uncommon methods that have at least been studied, such as synthesis into asynchronous systems built of threshold gates. And some, such as the homogeneous mixture of memory and logic, or the specification of wiring by genetic programs, we do not understand well enough to speculate on how the corresponding physical design algorithms might work.

None of these problems seems insurmountable, though none are easy. Physical design of biological systems has a long and interesting future ahead.

\section{ACKNOWLEDGMENTS}

To Ian Meinertzhagen and other biologists at the Janelia Campus of HHMI, for patiently tolerating my constant attempts to put a EE spin on biological problems. Funding provided by the Howard Hughes Medical Institute (HHMI).

\section{REFERENCES}

[1] Ryan Arvidson, Maayan Kaiser, Sang Soo Lee, Jean-Paul Urenda, Christopher Dail, Haroun Mohammed, Cebrina Nolan, Songqin Pan, Jason E Stajich, Frederic Libersat, et al. 2019. Parasitoid jewel wasp mounts multipronged neurochemical attack to hijack a host brain. Molecular \& Cellular Proteomics 18, 1 (2019), 99-114.

[2] Franc Brglez, David Bryan, and Krzysztof Kozminski. 1989. Combinational profiles of sequential benchmark circuits. In IEEE International Symposium on Circuits and Systems,. IEEE, 1929-1934.

[3] Silvia Budday, Paul Steinmann III, and Ellen Kuhl. 2015. Physical biology of human brain development. Frontiers in cellular neuroscience 9 (2015), 257.

[4] Alexandre Bystrov and Alexandre Yakovlev. 2002. Asynchronous circuit synthesis by direct mapping: Interfacing to environment. In Proceedings Eighth International Symposium on Asynchronous Circuits and Systems. IEEE, 127-136.

[5] Leigh E Chadwick. 1947. The respiratory quotient of Drosophila in flight. The Biological Bulletin 93, 3 (1947), 229-239.

[6] M Eugenia Chiappe, Johannes D Seelig, Michael B Reiser, and Vivek Jayaraman. 2010. Walking modulates speed sensitivity in Drosophila motion vision. Current Biology 20, 16 (2010), 1470-1475.

[7] Shamik Das, Anantha P Chandrakasan, and Rafael Reif. 2004. Calibration of Rent's rule models for three-dimensional integrated circuits. IEEE Transactions on Very Large Scale Integration (VLSI) Systems 12, 4 (2004), 359-366.

[8] Michael S Engel and David A Grimaldi. 2004. New light shed on the oldest insect. Nature 427, 6975 (2004), 627.

[9] Jonathan Green, Atsuko Adachi, Kunal K Shah, Jonathan D Hirokawa, Pablo S Magani, and Gaby Maimon. 2017. A neural circuit architecture for angular integration in Drosophila. Nature 546, 7656 (2017), 101.

[10] Paul H Harvey and John R Krebs. 1990. Comparing brains. Science 249, 4965 (1990), 140-146.

[11] Stanley Heinze. 2017. Unraveling the neural basis of insect navigation. Current opinion in insect science 24 (2017), 58-67.

[12] AL Hodgkin and AF Huxley. 1952. A Quantitative Description of Membrane Current and its Application to Conduction and Excitation in Nerve. F. Physiol 117 (1952), 500-544

[13] Kei Ito, Kazunori Shinomiya, Masayoshi Ito, J Douglas Armstrong, George Boyan, Volker Hartenstein, Steffen Harzsch, Martin Heisenberg, Uwe Homberg, Arnim Jenett, et al. 2014. A systematic nomenclature for the insect brain. Neuron 81, 4 (2014), 755-765.

[14] Thomas M Jessell and Joshua R Sanes. 2000. Development: The decade of the developing brain. Current opinion in neurobiology 10, 5 (2000), 599-611.
[15] Eric R Kandel, James H Schwartz, Thomas M Jessell, Department of Biochemistry, Molecular Biophysics Thomas Jessell, Steven Siegelbaum, and AJ Hudspeth. 2000. Principles of neural science. Vol. 4. McGraw-hill New York.

[16] Smita Krishnaswamy, Haoxing Ren, Nilesh Modi, and Ruchir Puri. 2009. DeltaSyn: An efficient logic difference optimizer for ECO synthesis. In 2009 IEEE/ACM International Conference on Computer-Aided Design-Digest of Technical Papers. IEEE, 789-796.

[17] Mary Yvonne Lanzerotti, Giovanni Fiorenza, and Rick A Rand. 2005. Microminiature packaging and integrated circuitry: The work of EF Rent, with an application to on-chip interconnection requirements. IBM journal of research and development 49, 4.5 (2005), 777-803.

[18] Matthias Meier and Alexander Borst. 2019. Extreme Compartmentalization in a Drosophila Amacrine Cell. Current Biology 29, 9 (2019), 1545-1550.

[19] Kathryn E Neville, Timothy L Bosse, Mia Klekos, John F Mills, Steven E Weicksel, James S Waters, and Marla Tipping. 2017. A novel ex vivo method for measuring whole brain metabolism in model systems. fournal of neuroscience methods (2017)

[20] Diogo Pimentel, Jeffrey M Donlea, Clifford B Talbot, Seoho M Song, Alexander JF Thurston, and Gero Miesenböck. 2016. Operation of a homeostatic sleep switch. Nature 536, 7616 (2016), 333.

[21] Margaret E Rice, Jyoti C Patel, and Stephanie J Cragg. 2011. Dopamine release in the basal ganglia. Neuroscience 198 (2011), 112-137.

[22] Louis K Scheffer, C Shan Xu, Michal Januszewski, Zhiyuan Lu, Shin-ya Takemura, Kenneth J Hayworth, Gary Huang, Kazunori Shinomiya, Jeremy Maitlin-Shepard, Stuart Berg, et al. 2020. A Connectome and Analysis of the Adult Drosophila Central Brain. ELife (2020), e57443. https://doi.org/10.7554/eLife.57443

[23] I Segev, JW Fleshman, JP Miller, and B Bunow. 1985. Modeling the electrical behavior of anatomically complex neurons using a network analysis program: passive membrane. Biological cybernetics 53, 1 (1985), 27-40.

[24] Kazunori Shinomiya, Shin-ya Takemura, Patricia K Rivlin, Stephen M Plaza, Louis K Scheffer, and Ian A Meinertzhagen. 2015. A common evolutionary origin for the $\mathrm{ON}$-and OFF-edge motion detection pathways of the Drosophila visual system. Frontiers in Neural Circuits 9 (2015), 33.

[25] Nicholas James Strausfeld. 2009. Brain organization and the origin of insects: an assessment. Proceedings of the Royal Society B: Biological Sciences 276, 1664 (2009), 1929-1937.

[26] Richard M Swanson and James D Meindl. 1972. Ion-implanted complementary MOS transistors in low-voltage circuits. IEEE Journal of Solid-State Circuits 7, 2 (1972), 146-153.

[27] Shin-ya Takemura, Yoshinori Aso, Toshihide Hige, Allan Wong, Zhiyuan Lu, C Shan Xu, Patricia K Rivlin, Harald Hess, Ting Zhao, Toufiq Parag, et al. 2017. A connectome of a learning and memory center in the adult Drosophila brain. Elife 6 (2017).

[28] Shin-ya Takemura, Arjun Bharioke, Zhiyuan Lu, Aljoscha Nern, Shiv Vitaladevuni, Patricia K Rivlin, William T Katz, Donald J Olbris, Stephen M Plaza, Philip Winston, et al. 2013. A visual motion detection circuit suggested by Drosophila connectomics. Nature 500, 7461 (2013), 175.

[29] Fabian David Tschopp, Michael B Reiser, and Srinivas C Turaga. 2018. A connectome based hexagonal lattice convolutional network model of the Drosophila visual system. arXiv preprint arXiv:1806.04793 (2018).

[30] Daniel B Turner-Evans, Kristopher Jensen, Saba Ali, Tyler Paterson, Arlo Sheridan, Robert P Ray, Scott Lauritzen, Davi Bock, and Vivek Jayaraman. 2019. The neuroanatomical ultrastructure and function of a biological ring attractor. bioRxiv (2019), 847152

[31] John C Tuthill, Aljoscha Nern, Stephen L Holtz, Gerald M Rubin, and Michael B Reiser. 2013. Contributions of the 12 neuron classes in the fly lamina to motion vision. Neuron 79, 1 (2013), 128-140.

[32] Brett A Wagner, Sujatha Venkataraman, and Garry R Buettner. 2011. The rate of oxygen utilization by cells. Free Radical Biology and Medicine 51, 3 (2011), $700-712$.

[33] Xiaojian Yang, Elaheh Bozorgzadeh, and Majid Sarrafzadeh. 2001. Wirelength estimation based on Rent exponents of partitioning and placement. In Proceedings of the 2001 international workshop on System-level interconnect prediction. ACM, 25-31.

[34] Rui Zhang, Pallav Gupta, Lin Zhong, and Niraj K Jha. 2004. Threshold network synthesis and optimization and its application to nanotechnologies. IEEE Transactions on computer-aided design of integrated circuits and systems 24, 1 (2004), 107-118. 